\title{
PAPER
}

\section{Effects of deep brain stimulation and levodopa on postural sway in Parkinson's disease}

\author{
L Rocchi, L Chiari, F B Horak
}

J Neurol Neurosurg Psychiatry 2002;73:267-274

See end of article for authors' affiliations ......................

Correspondence to: Dr F B Horak, Neurological Sciences Institute, Oregon Health \& Science

University, 505 NW 185th Avenue, Beaverton, OR 97006-3499, USA;

horak@@ohsu.edu

Received

24 January 2002

In revised form 16 May

2002

Accepted 20 May 2002

\begin{abstract}
Objective: To quantify postural sway in subjects with Parkinson's disease and elderly controls, and determine the effects of Parkinson's disease, deep brain stimulation, levodopa, and their interactions on postural control during quiet stance.

Methods: Centre of foot pressure (CoP) displacement under each foot was measured during three 60 $s$ trials of quiet stance with eyes open in 11 controls and six patients with Parkinson's disease. Subjects with Parkinson's disease were tested in four treatment conditions: off both deep brain stimulation and levodopa (off condition); on deep brain stimulation; on levodopa; and on both deep brain stimulation and levodopa. The variables extracted from CoP included: root mean square distance (rms), mean velocity, $95 \%$ power frequency $\left(f_{95 \%}\right)$, area of the $95 \%$ confidence ellipse (ellipse area), direction of its major axis (mdir), and postural asymmetry between the feet.

Results: rms and area of postural sway were larger than normal in subjects with Parkinson's disease in the off condition, increased further with levodopa, and significantly decreased with deep brain stimulation. Mean velocity and $f_{95 \%}$ were also larger than normal but were restored to normal by all treatments, especially by deep brain stimulation. The combined effect of deep brain stimulation and levodopa resulted in a postural sway that was an average of the effect of each treatment individually. Levodopa increased sway more in the mediolateral than in the anterior-posterior direction. Subjects with Parkinson's disease had asymmetrical mean velocity and $f_{95 \%}$ between the feet, and this asymmetry increased with levodopa but decreased with deep brain stimulation. The $f_{95 \%}$ of the CoP correlated with tremor, posture, and gait subcomponents of the unified Parkinson's disease rating scale.

Conclusions: Subjects with Parkinson's disease have abnormal postural sway in stance. Treatment with levodopa increases postural sway abnormalities, whereas treatment with deep brain stimulation improves postural sway. Quantitative evaluation of static posturography may be a useful adjunct to clinical measures in patients with Parkinson's disease.
\end{abstract}

mpairment of postural control is a major problem in patients with Parkinson's disease, and postural problems become increasingly severe as the disease progresses, despite treatment with levodopa. ${ }^{2}$ This increasing severity may reflect the increased involvement of non-dopaminergic pathways, which results in the declining effectiveness of levodopa replacement therapy. ${ }^{3}$ There is new hope that an alternative treatment for Parkinson's disease involving high frequency deep brain stimulation of the subthalamic nucleus or globus pallidus may be more effective than levodopa in improving postural control, presumably because deep brain stimulation also affects non-dopaminergic pathways. ${ }^{35}$

Most studies that have examined the effectiveness of levodopa and deep brain stimulation in treating the motor control problems of Parkinson's disease, such as postural instability, have used clinical rating scales, for example the unified Parkinson's disease rating scale (UPDRS). Such measures of postural control are not designed to differentiate among underlying causes of postural instability, ${ }^{67}$ and they suffer from poor sensitivity for detecting postural problems. ${ }^{8}$ For example, these tests cannot determine whether patients with Parkinson's disease have poor stability because they allow their centre of mass to move too far or too fast, or whether rigidity, bradykinesia, or tremor limits their ability to control their centre of mass. In addition, the one item on the UPDRS that specifically focuses on postural stability-the retropulsion test-is not well correlated with quantitative measures of a patient's ability to recover from an external disturbance. ${ }^{8}$

In the present study we quantified postural sway in subjects with Parkinson's disease, in order to understand the effect of Parkinson's disease on postural control, to investigate changes in postural control resulting from deep brain stimulation and levodopa, and their interaction, and to determine whether quantitative changes in postural sway caused by treatment are reflected in changes in UPDRS measures of postural control.

Few studies have quantified postural stability during quiet stance in subjects with Parkinson's disease. In those that did, the results were often contradictory and limited. Some studies did not find abnormalities, ${ }^{9}$ while others found less postural sway in subjects with Parkinson's disease than in aged matched control subjects. ${ }^{10}$ Such discrepancies may be explained by differences in research design: subject populations in different studies had different severities of Parkinson's disease, and some subject populations were on levodopa while others were off. In only one study was the effect of levodopa treatment on postural sway examined, and in that study only the anteroposterior direction was considered; a change in sway velocity was found but not in sway area. ${ }^{11}$ However, another study suggested that, when on levodopa, subjects with Parkinson's disease have more difficulty in controlling lateral postural sway than anteroposterior sway. ${ }^{12}$ Studies have also suggested that increased lateral sway is associated with an increased risk of falling in elderly subjects. ${ }^{13} 14$

The results of previous studies in our laboratory suggest that automatic postural control may involve basal ganglia circuits distinct from those controlling voluntary postural movement; for example, levodopa is more effective at improving

Abbreviations: CoP, centre of foot pressure; dbs, deep brain stimulation; $f_{95 \%}$, frequency below which $95 \%$ of the total power is found; UPDRS, unified Parkinson's disease rating scale 
Table 1 Demographic and clinical data of subjects with Parkinson's disease

\begin{tabular}{|c|c|c|c|c|c|c|c|c|c|c|c|c|}
\hline \multirow[b]{2}{*}{ Subject } & \multirow[b]{2}{*}{ DBS site } & \multirow[b]{2}{*}{$\begin{array}{l}\text { Age } \\
\text { (years) }\end{array}$} & \multirow[b]{2}{*}{$\begin{array}{l}\text { PD duration } \\
\text { (years) }\end{array}$} & \multicolumn{4}{|c|}{ UPDRS } & \multicolumn{4}{|c|}{ Dyskinesia scores } & \multirow[b]{2}{*}{ Anti-PD drugs } \\
\hline & & & & OFF & DBS & DOPA & $\begin{array}{l}\text { DOPA } \\
+ \text { +DBS }\end{array}$ & OFF & DBS & DOPA & $\begin{array}{l}\text { DOPA } \\
+ \text { DBS }\end{array}$ & \\
\hline$I^{(1)}$ & STN & 61 & 18 & 75 & 32 & 33 & 25 & 0 & 2 & 5 & 7 & $\begin{array}{l}\text { Carbi/Levodopa, atenolol, } \\
\text { levothyroxine }\end{array}$ \\
\hline$\|$ & GPi & 48 & 11 & 41 & 40 & 30.5 & 28 & 0 & 0 & 0 & 3 & $\begin{array}{l}\text { Carbi/Levodopa, } \\
\text { bromocriptine, } \\
\text { trihexythenidyl }\end{array}$ \\
\hline III & STN & 53 & 21 & 85 & 61 & 25.5 & 25 & 0 & 0 & 0 & 3 & $\begin{array}{l}\text { Carbi/Levodopa, } \\
\text { trihexynidyl }\end{array}$ \\
\hline IV & GPi & 75 & 8 & 40 & 32 & 26.5 & 22 & 0 & 0 & 5 & 0 & $\begin{array}{l}\text { Carbi/Levodopa, } \\
\text { amantadine }\end{array}$ \\
\hline v & GPi & 61 & 12 & 21.5 & 10 & 12.5 & 5.5 & 0 & 0 & 0 & 0 & $\begin{array}{l}\text { Carbi/Levodopa, } \\
\text { amantadine, pergolide, } \\
\text { Flomax, Zoloft }\end{array}$ \\
\hline VI & STN & 69 & 19 & 78 & 50 & 19 & 11.5 & 0 & 0 & 0 & 0 & Carbi/Levodopa, pergolide \\
\hline
\end{tabular}

(1)Dyskinesia scores collected one year after surgery instead of six months.

DBS, deep brain stimulation; DOPA, dopamine treatment; GPi, globus pallidus internus; OFF, no treatment; STN, subthalamic nucleus.

voluntary postural movements-such as step initiation and rise to toes-than automatic postural responses to external perturbations. ${ }^{1516}$ Because levodopa is more effective for voluntary than for automatic postural control in subjects with Parkinson's disease, we hypothesised the following:

- that levodopa is not effective in improving control of quiet stance;

- that deep brain stimulation is more effective than levodopa for automatic postural control ${ }^{17}$ because deep brain stimulation affects both dopaminergic and non-dopaminergic pathways;

- that the interaction of deep brain stimulation with levodopa results in improved postural stability.

\section{METHODS}

\section{Subjects}

Six subjects with Parkinson's disease and 11 healthy control subjects gave informed consent before inclusion in this study, approved by the human subject committee of Good Samaritan Hospital, Portland, Oregon.

Control subjects were physically active and without musculoskeletal or neurological disorders. Their mean (SD) age was 64 (6.4) years (range 54 to 74 years). The mean age of the subjects with Parkinson's disease was 61 (9.1) years (range 48 to 75 years), and the duration of their disease, 16 (5.4) years (range 8 to 21 years). All subjects with Parkinson's disease could stand independently and were responsive to levodopa, but fluctuated excessively between being on and off levodopa. Medical examination and history showed they did not have other pathological conditions that could affect postural control.

Table 1 presents ages, duration of Parkinson's disease, total motor UPDRS scores, clinical dyskinesia scores, and drugs taken. All subjects with Parkinson's disease were scored 2.5-5 in the Hoehn and Yahr scales, depending on treatment condition.

The patients with Parkinson's disease were a subset of subjects from a randomised double blinded clinical study investigating deep brain stimulation in the subthalamic nucleus or globus pallidus (see Burchiel et al for details of surgery and optimisation of stimulation parameters ${ }^{18}$ ). During surgery bilateral electrodes were implanted either in the subthalamic nucleus or in the globus pallidus under local anaesthesia. Subjects with electrodes implanted bilaterally in the subthalamic nucleus or globus pallidus were not differentiated in this study because the small number of subjects did not allow comparative analyses.

Subjects with Parkinson's disease were tested six months after surgery to allow the effects of deep brain stimulation to stabilise. ${ }^{18}$ They were tested under four treatment conditions:
- Baseline (that is, off levodopa and off stimulation (off condition));

- Only deep brain stimulation (dbs condition);

- Only drug treatment (dopa condition);

- Both deep brain stimulation and levodopa (dbs+dopa condition).

The off condition was obtained by a dopamine washout of at least 12 hours and shutting the stimulator off 20 minutes before tests. The subjects were considered off or on treatment by clinical signs including change in the UPDRS, $0-10$ self rating scale, and minimal dyskinesia. Conditions were tested in the following order: (1) dbs (approximately 40 minutes after turning stimulators on); (2) off; (3) dopa (approximately 60 minutes after administration of optimised dopamine treatment); (4) $d b s+d o p a$. The UPDRS motor examination (score 0-108) and dyskinesia scores (0-4 summed for each arm and leg, and head for a range of 0-20) were acquired in all four conditions just before posture testing.

\section{Static posturography}

To measure postural sway, subjects stood on a dual force plate platform, with one foot on each force plate, for three sequential trials. Subjects were instructed to maintain an upright standing position, with arms at their sides, eyes open with gaze straight ahead at an art poster, and feet shoulder width apart. Four vertical forces were recorded from each force plate at $480 \mathrm{~Hz}$ for 60 seconds, and data were filtered by a 30th order low pass FIR digital filter (cut off frequency $10 \mathrm{~Hz}$ ) and downsampled at $20 \mathrm{~Hz}$.

The centre of foot pressure $(\mathrm{CoP})$ of each foot $\left(\mathrm{CoP}_{\text {left }}\right.$ and $\mathrm{CoP}_{\text {right }}$ ) and the total body $\mathrm{CoP}$ were computed from the vertical forces. The CoP is the point location of the ground reaction force vector ${ }^{19}$ and reflects the sway of the body (biological noise) and forces used to maintain the centre of gravity within the support base. ${ }^{2021}$

\section{CoP postural sway variables}

Three uncorrelated CoP variables characterised postural steadiness $^{20}$ : (1) root mean square distance ( $\mathrm{rms}$ ), which quantifies the CoP variability around the mean CoP trajectory; (2) mean velocity of CoP displacement; and (3) 95\% power frequency $\left(f_{95 \%}\right)$, which represents the frequency below which $95 \%$ of the total power is found. These variables were computed for the bidimensional CoP trajectory (both the anteroposterior and mediolateral directions of total body CoP displacement), and for individual foot CoP displacements. In addition, a 95\% confidence ellipse for each trial was estimated, ${ }^{20}$ which encloses approximately $95 \%$ of the points on the CoP trajectory. The area of the confidence ellipse 


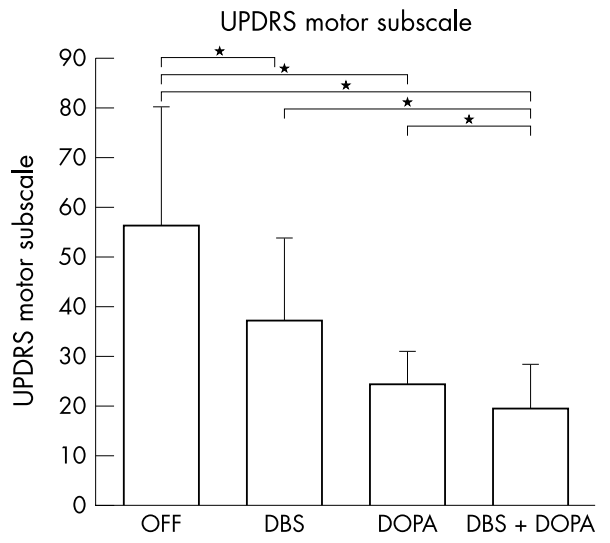

Figure 1 Mean values of the UPDRS motor examination (range $0-108$ ) in subjects with Parkinson's disease in the four conditions. DBS, under deep brain stimulation only; DBS+DOPA, under deep brain stimulation and levodopa; DOPA, under levodopa only; OFF, no treatment; UPDRS, unified Parkinson's disease rating scale. Error bars $=S D .{ }^{*} p<0.05$.

(ellipse area) and the direction of maximum sway (mdir) were quantified.

Comparisons of CoP variables under each foot allowed measures of postural symmetry. Postural symmetry was examined using the absolute symmetry index. ${ }^{23}$ If $\mathrm{P}_{\text {left }}$ and $\mathrm{P}_{\text {right }}$ are values of a parameter extracted from $\mathrm{CoP}_{\text {left }}$ and $\mathrm{CoP}_{\text {right }}$ the absolute symmetry index ( $\mathrm{ASI}_{\mathrm{p}}$ ) for this parameter is defined as:

$$
\operatorname{ASI}_{p}=\left|\frac{2 \cdot\left(P_{\text {left }}-P_{\text {right }}\right)}{P_{\text {left }}+P_{\text {right }}}\right| \cdot 100
$$

\section{Statistical analysis}

The non-parametric Wilcoxon signed-rank test ${ }^{24}$ compared UPDRS motor scores and differences among sway parameters in subjects with Parkinson's disease under the four conditions. Analysis of variance (ANOVA) (repeated measures) compared variables between control subjects and subjects with Parkinson's disease. Correlation analysis between CoP and UPDRS subscores was performed across all four conditions with the non-parametric Spearman's rank method. ${ }^{24}$

\section{RESULTS}

Clinical scores

Results from the UPDRS motor examination for each condition are represented in fig 1 . In the off condition, the mean UPDRS motor score was largest (worst clinical signs), and the standard deviation was also largest. The UPDRS score was significantly reduced in the $d b s$ and dopa conditions. The decrease was larger in the dopa condition than in the $d b s$ condition. In the $d b s+d o p a$ condition, UPDRS scores were further reduced compared with the dopa and $d b s$ conditions. The variability also decreased under treatment, especially in the dopa condition.

\section{General characteristics of postural sway}

The CoP trajectory of a representative control subject is illustrated in fig 2. Postural sway displacement of control subjects was larger in the anteroposterior than in the mediolateral direction. ${ }^{19}$

In fig 3, CoP trajectories are shown during each condition for a representative subject with Parkinson's disease. Both Parkinson's disease and the different treatment conditions significantly affected postural sway. In the off condition (fig $3 \mathrm{~A})$, although the range of CoP displacement was apparently similar to the control subject in fig 2, its shape was quite different, with frequent changes of direction. On the other hand, in the $d b s$ condition (fig $3 \mathrm{~B}$ ), postural sway looked normal in shape and even slightly smaller than normal in range. In the dopa condition (fig 3C), the main features of the CoP trajectory changed. The CoP trajectory covered a larger area with a larger than normal range of sway especially in the mediolateral direction, in which it was almost as large as in the anteroposterior direction. Postural sway in the dbs+dopa condition (fig 3D) was more similar to the postural sway in the dopa condition than in the $d b s$ condition.

\section{Measures of postural stability in standing}

Group means of total body CoP variables are summarised in fig 4 for rms, mean velocity, and $\mathrm{f}_{95 \%}$. In the off condition, subjects with Parkinson's disease showed larger rms (fig 4A) than control subjects. Deep brain stimulation reduced rms $(p<0.05)$ to within normal limits. The intersubject variability of rms was also significantly reduced in the $d b s$ condition compared with the off condition. Conversely, dopamine increased rms of CoP displacement, making it more than twice the value of control subjects $(\mathrm{p}<0.01)$. In the dopa condition rms was also significantly larger than in the $d b s$ condition
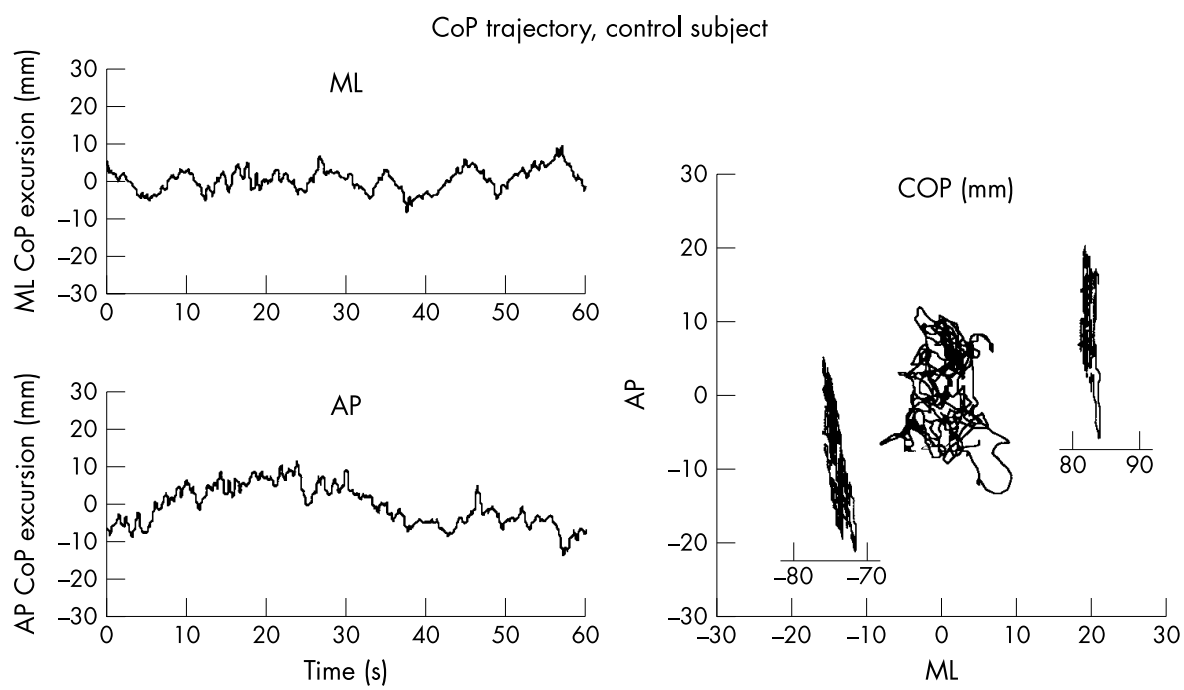

Figure 2 Centre of foot pressure (CoP) trajectory of a representative control subject. Left: Monodimensional time series in mediolateral (ML) and anteroposterior (AP) directions, obtained from total body CoP. Right: Bidimensional displacement of CoP sway in the horizontal plane of each single foot and of the total body (centre). For simplicity, the time series are normalised to their mean value. 
CoP trajectory, subject with Parkinson's disease
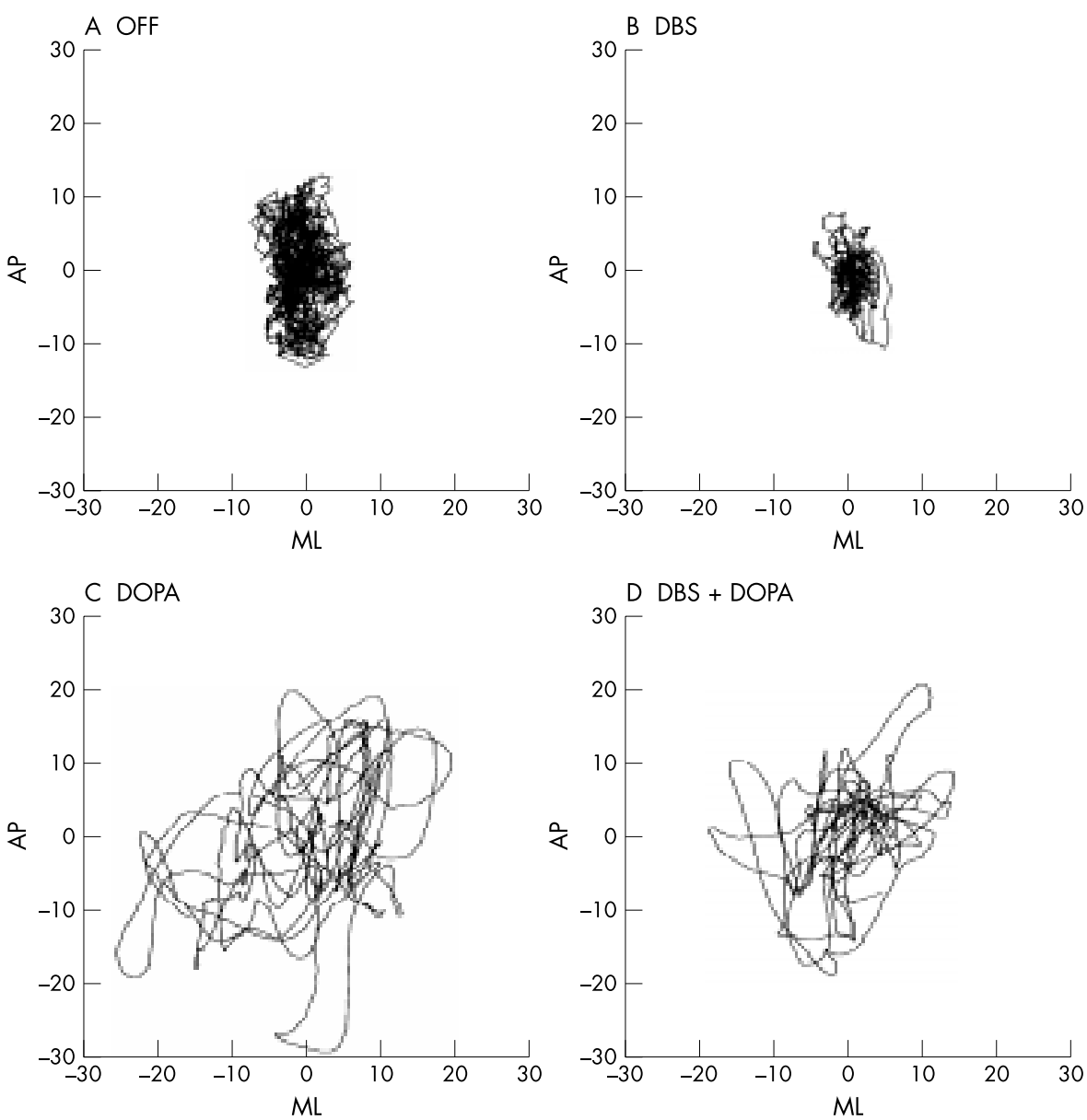

Figure 3 Centre of foot pressure (CoP) trajectories in the horizontal plane of a representative subject with Parkinson's disease in the four test conditions (panels $A$ to $D$ ). For simplicity, the time series are normalised to their mean value. DBS, under deep brain stimulation only; DBS+DOPA, under deep brain stimulation and levodopa; DOPA, under levodopa only; OFF, no treatment.

$(\mathrm{p}<0.01)$. In the $d b s+$ dopa condition, rms remained high and almost unchanged compared with the off condition.

Mean velocity of CoP displacement (fig 4B) was also strongly affected by Parkinson's disease and the dopa and $d b s$ conditions. The largest mean velocity occurred in the off condition, in which it was about eight times the control subjects' values $(p<0.05)$. This large mean velocity reflects the long sway path travelled over 30 seconds by subjects with Parkinson's disease. In all treatment conditions, mean velocity was decreased compared with the off condition, even if statistical significance was only approached because of the large intersubject variability in the off condition. However, in the $d b s$ condition, mean velocity of subjects with Parkinson's disease was very close to mean velocity of control subjects $(p>0.05)$,
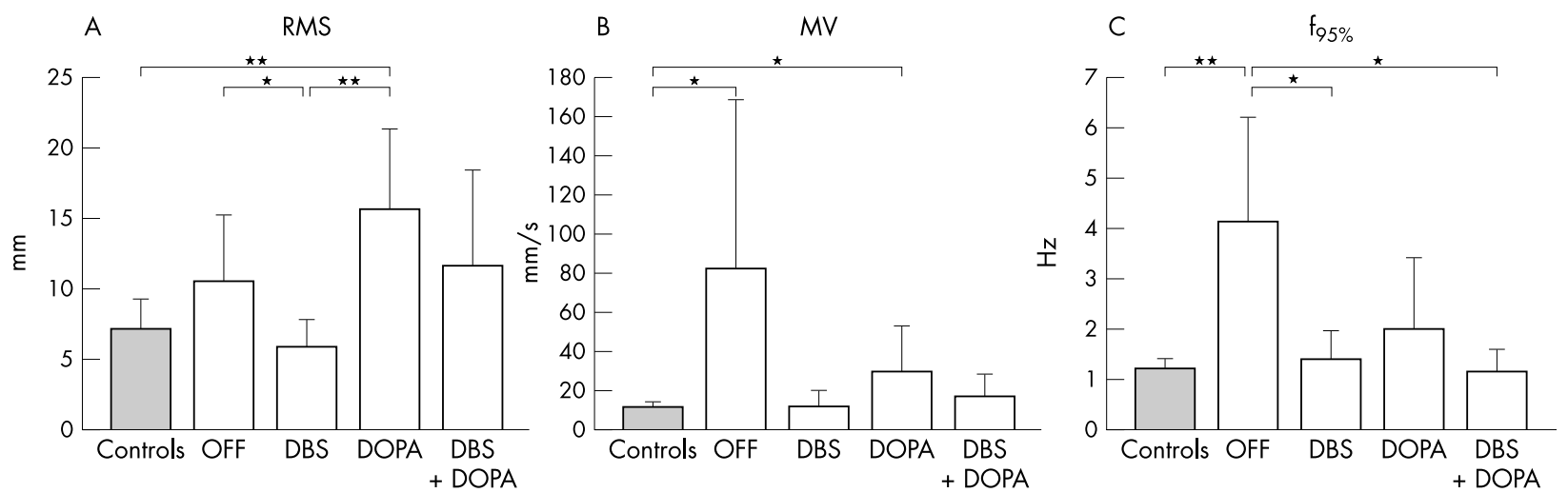

Figure 4 Variables extracted from bidimensional centre of foot pressure (CoP) excursions. (A) Root mean square (rms) of distance of CoP from the centre of sway. (B) Mean velocity (MV) of CoP trajectory. (C) Frequency below which lie the $95 \%$ of the total power $\left(\mathrm{f}_{95 \%}\right)$. For each variable, bars represent means and the error bars, 1 SD. Control subjects are represented by shaded bars, subjects with Parkinson's disease by empty bars. DBS, under deep brain stimulation only; DBS+DOPA, under deep brain stimulation and levodopa; DOPA, under levodopa only; OFF, no treatment. ${ }^{*} p<0.05 ;{ }^{* *} p<0.01$. 


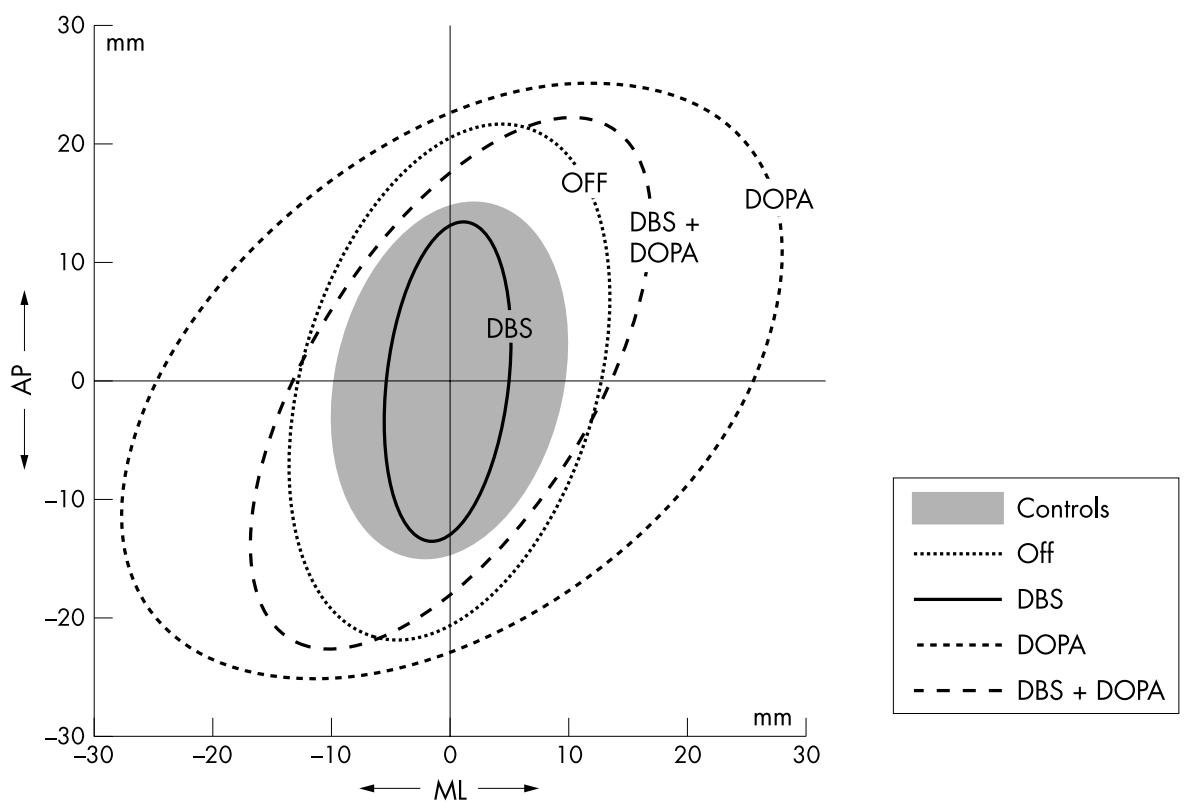

Figure 5 Mean 95\% confidence ellipses, computed from bidimensional centre of foot pressure excusions of control subjects and subjects with Parkinson's disease in the four test conditions. AP, anteroposterior; DBS, under deep brain stimulation only; DBS+DOPA, under deep brain stimulation and levodopa; DOPA, under levodopa only; ML, mediolateral; OFF, no treatment.

whereas in the dopa condition, mean velocity for subjects with Parkinson's disease remained three times larger than for control subjects $(\mathrm{p}<0.05)$. The combined effect of both treatments (dbs+dopa condition) resulted in a mean velocity that was an average of the values measured under each treatment individually, producing a mean velocity of less than twice the normal value.

Control subjects' $\mathrm{f}_{95 \%}$ was consistent, with a mean value of $1.23 \mathrm{~Hz}$ (fig 4C) - that is, on average, only $5 \%$ of the total CoP power was spread over frequencies higher than $1.23 \mathrm{~Hz}$. Thus it was rare to find $\mathrm{CoP}$ oscillations with a time period shorter than 0.8 seconds in control subjects. In the off condition, subjects with Parkinson's disease showed a significantly larger $\mathrm{f}_{95 \%}$ (mean $4.08 \mathrm{~Hz})$ than control subjects $(\mathrm{p}<0.01)$. Such an increase in CoP frequency bandwidth could be related to the presence of postural tremor, which has a typical frequency of about 4-5 Hz in the CoP signal. ${ }^{1125}$ All treatment conditions $(d o p a, d b s, d b s+d o p a)$ restored $f_{95 \%}$ values closer to the values for control subjects. The two $d b s$ conditions significantly reduced the $\mathrm{f}_{95 \%}$ compared with the off condition $(\mathrm{p}<0.05)$. In fact, $\mathrm{f}_{95 \%}$ was not significantly different between the control subjects and subjects with Parkinson's disease in the $d b s$ and $d b s+d o p a$ conditions $(\mathrm{p}>0.05)$. Although decreased compared with the off condition, $\mathrm{f}_{95 \%}$ for the dopa condition (mean $2 \mathrm{~Hz}$ ) remained higher than control values.

The group mean $95 \%$ confidence ellipses representing the CoP trajectories for each condition are shown in fig 5 . The group mean values and ranges of ellipse area and orientation (mdir) are reported in table 2. Control subjects' mdir was, on average, close to $80^{\circ}$ - that is, postural sway was primarily in the anteroposterior direction. For subjects with Parkinson's disease in the off condition, ellipse area was larger than normal owing to an increase of sway mostly in the anteroposterior direction, such that mdir remained around $70-80^{\circ}$. In the $d b s$ condition, ellipse area was greatly reduced and was even smaller than for control subjects, and the mdir was maintained close to $90^{\circ}$. Overall, in the $d b s$ condition, the CoP 95\% confidence ellipse was closer to normal than in the other conditions. In the dopa condition, ellipse area was larger than normal $(\mathrm{p}<0.01)$, as illustrated in fig 5 , and mdir shifted toward the mediolateral direction (mean value $38.6^{\circ}$ ), which was significantly different from normal values $(\mathrm{p}<0.01)$. In the $d b s+d o p a$ condition, postural sway was reduced in the mediolateral direction, and mdir moved toward the anteroposterior direction compared with the dopa condition. Nevertheless, the CoP ellipse values for subjects with Parkinson's disease in the dbs+dopa condition remained different from control subjects.

\section{Postural asymmetry}

A surprising degree of asymmetry in postural control between the feet during stance was observed in the elderly control subjects (table 3). The rms asymmetry in subjects with Parkinson's disease across the four conditions was never much higher than for control subjects (with the highest values in the off condition and the lowest values in the $d b s$ condition; table $3)$. These results suggest that neither Parkinson's disease nor treatment conditions significantly affected the difference in size of the left and right CoP displacement.

In contrast, the mean velocity and $\mathrm{f}_{95 \%}$ were significantly more asymmetrical in subjects with Parkinson's disease (off condition) than in control subjects, indicating the dominance of one extremity in controlling the velocity and frequency of postural sway. This asymmetry was brought back to normal values by deep brain stimulation, probably owing to the bilateral nature of electrical stimulation. Results in the $d b s$ and

Table 2 Mean values and ranges of confidence ellipse area (Area) and maximum variance direction (MDir) for control subjects and subjects with Parkinson's disease in the four test conditions

\begin{tabular}{llllll}
\hline \multirow{2}{*}{ Condition } & \multicolumn{2}{l}{ Area $\left(\mathrm{mm}^{2}\right)$} & & \multicolumn{2}{l}{ MDir (degrees) } \\
\cline { 2 - 3 } \cline { 5 - 6 } \cline { 5 - 6 } & Mean & Range & & Mean & Range \\
\hline Controls & 484 & 197 to 998 & & 78 & 35 to 124 \\
OFF & 1044 & 290 to 2947 & & 73 & 10 to 100 \\
DBS & 246 & 72 to 685 & & 85 & 68 to 102 \\
DOPA & $2157^{\text {a,b }}$ & 1138 to 4075 & & $39^{\text {c,d }, e}$ & 3 to 79 \\
DBS+DOPA & 1179 & 287 to 3907 & & 57 & 11 to 103 \\
\hline
\end{tabular}

${ }^{a} \mathrm{p}<0.05 v \mathrm{DBS} ;{ }^{b} \mathrm{p}<0.01$ v controls; ${ }^{c} \mathrm{p}<0.05$ v OFF; ${ }^{\mathrm{d}} \mathrm{p}<0.01 v$ controls; ${ }^{e} \mathrm{p}<0.01 \vee$ DBS

DBS, deep brain stimulation; DOPA, dopamine treatment; OFF, on no treatment. 
Table 3 Asymmetry (ASI) index, computed from data obtained from each single plate of the double force platform

\begin{tabular}{llll}
\hline & ASI $_{\text {RMS }}$ & ASI $_{M V}$ & ASIf $_{95 \%}$ \\
\hline Controls & $23(10) \%$ & $27(13) \%$ & $8(6) \%$ \\
OFF & $29(19) \%$ & $38(31) \%$ & $28(40) \%$ \\
DBS & $25(11) \%$ & $27(9) \%$ & $10(7) \%$ \\
DOPA & $26(17) \%$ & $43(34) \%$ & $40(42) \%$ \\
DBS+DOPA & $30(26) \%$ & $24(19) \%$ & $14(9) \%$ \\
\hline
\end{tabular}

Values are mean (SD)

DBS, deep brain stimulation; DOPA, dopamine; $f_{95 \%}, 95 \%$ power frequency; $M V$, mean velocity; OFF, on no treatment; RMS, root mean square distance.

$d b s+$ dopa conditions were similar. The largest absolute symmetry index was obtained in the dopa condition, suggesting that levodopa may worsen asymmetry of stance control.

\section{Correlation with clinical scores}

Spearman's rank correlation coefficients indicated that the only CoP based postural variable that significantly correlated with the UPDRS or its components was $\mathrm{f}_{95 \%}$. The $\mathrm{f}_{95 \%}$ value correlated significantly with the global UPDRS motor examination score $(r=0.43 ; \mathrm{p}=0.04)$ and with overall severity of posture + gait (items 26-31; $r=0.49 ; \mathrm{p}=0.016$ ), in particular, with gait (item 29, $r=0.60 ; \mathrm{p}=0.003$ ), bradykinesia (item 31, $r=0.60, \mathrm{p}=0.003$ ), and tremor (items 20-21, $r=0.56$, $\mathrm{p}=0.024)$. The high correlations between $\mathrm{f}_{95 \%}$ and these components of UPDRS suggest that poor performance in axial motor tasks is associated with an increase in postural sway frequency. Interestingly, the posture component (item 28) and the postural stability (pull test; item 30) component of the UPDRS did not correlate with $\mathrm{f}_{95 \%}$ or with another quantitative CoP variable.

\section{DISCUSSION}

In this study we found that subjects with Parkinson's disease have abnormal postural sway in stance and that treatment with levodopa increases postural sway abnormalities, whereas treatment with deep brain stimulation improves postural sway. The differences in quantitative CoP measures between control subjects and subjects with Parkinson's disease in the off condition illustrate the way in which neural postural control of stance is affected by Parkinson's disease when no therapeutic intervention is provided. The high mean velocity of sway for subjects with Parkinson's disease in the off condition can be interpreted as the large amount of neural control activity needed by these patients to stabilise posture in stance. ${ }^{20}$

In addition to frequent corrections of postural sway, the rigidity and passive stiffness of Parkinsonian muscles and joints may be responsible for rapid, jerky CoP movements and frequent adjustments of the CoP trajectory characteristic of the off condition. Studies have shown that subjects with Parkinson's disease have higher passive stiffness of ankle joints in stance and show larger background EMG activity and more co-contraction of leg muscles in stance. ${ }^{1126}$ Stiffer systems sway at faster velocities and higher frequencies, so it is not surprising that subjects with Parkinson's disease in the off condition showed a high value of $\mathrm{f}_{95 \%}$. The higher than normal frequencies of sway in subjects with Parkinson's disease were probably related to postural tremor as well as to the short time between sequential CoP adjustments. Power spectral density plots showed an additional $4-5 \mathrm{~Hz}$ added power in Parkinson's disease subjects off dopamine, and $\mathrm{f}_{95 \%}$ values were significantly correlated with resting tremor in the UPDRS, consistent with the 4-5 $\mathrm{Hz}$ body tremor characteristic of people with Parkinson's disease. ${ }^{11}$ In fact, although tremor in the arms may be weakly reflected in oscillations in the CoP during stance, studies have shown that many people with Parkinson's disease also have tremors in leg and trunk muscles that are not always obvious upon clinical examination. ${ }^{1127}$

In spite of the frequent neural control over the CoP by subjects with Parkinson's disease, their CoP displacement was larger than normal, as measured by rms and area. This large area of postural sway reflects the ineffectiveness of postural control and deficits in fine tuning movements that may be related to poor use of somatosensory information in this condition. Several studies have shown that people with Parkinson's disease have problems in sensitively detecting kinaesthetic and proprioceptive information. ${ }^{28-31}$

Somatosensory information from the feet, legs, and trunk is the most critical sensory information for control of posture during standing. ${ }^{32-34}$ The large postural sway area may reflect delayed somatosensory feedback in the postural control loop and inaccurate, noisy detection of the centre of mass position. Studies have shown that when somatosensory feedback for posture is delayed peripherally owing to peripheral neuropathy, multiple sclerosis, or dorsal root afferent degeneration, the CoP drifts, resulting in larger than normal rms, ellipse area, and mean velocity of $\mathrm{CoP}^{32}{ }^{35-37}$ The increased postural sway area in people with Parkinson's disease is probably not caused by loss of peripheral somatosensory information associated with aging, because sway area was much larger in the age matched control subjects; in addition, clinical examination showed no more loss of proprioceptive sensation in the feet of subjects with Parkinson's disease than in the controls.

Our results also suggest that deep brain stimulation and levodopa affect postural control of stance very differently in patients with Parkinson's disease. Our finding that postural stability worsened in the dopa condition is consistent with the clinical observation that there is no significant improvement in balance with levodopa treatment. ${ }^{38} 3940$ The area of postural sway may increase on levodopa compared with the off condition because of the presence of dyskinesia in the dopa condition. However, dyskinesia is probably not the primary factor accounting for the increased sway area in this study because the levels of dyskinesia were very low or non-existent during testing (table 1), and the clinical dyskinesia rating scale was not significantly correlated with rms or the area of the $95 \%$ confidence ellipse. Dyskinesia is also unlikely to account for the increased postural sway area in the dopa condition because mean velocity and $\mathrm{f}_{95 \%}$ did not increase with the rms, as would be expected by an increase in frequency of involuntary, quick dyskinetic movements. Ellipse area more probably increased from the off to the dopa condition because postural tone decreased without a concomitant improvement in postural control. A decrease in tone caused by levodopa is consistent with the decrease in mean velocity and $\mathrm{f}_{95 \%}$ in the dopa condition compared with the off condition. ${ }^{11}$ A decrease in stiffness in the dopa conditions would allow the CoP to move even further than the large sway, owing to poor or delayed somatosensory feedback. Similar worsening of postural control with levodopa caused by decreased stiffness without improved postural control was observed in automatic postural responses of subjects with Parkinson's disease. ${ }^{16}$ A recent study suggests that levodopa does not improve proprioceptive and kinaesthetic deficits, consistent with the lack of improvement in postural sway in the dopa condition in our study. ${ }^{31}$

Levodopa increased postural sway primarily in the mediolateral direction. The large mediolateral sway may reflect a decrease in postural tone at the trunk and hip levels, which would decouple the trunk from lower limb sway. ${ }^{12}$ Postural control of the trunk has been shown to be primarily in the mediolateral direction during gait. ${ }^{41}$ Risk of falling has been found to correlate with a large lateral sway during stance in the elderly. ${ }^{14}$ Studies are needed to compare the incidence of falls and quantitative postural sway measures with a longitudinal analysis on subjects with Parkinson's disease who are being treated with levodopa. 
Deep brain stimulation appears to be effective in stabilising stance, as indicated by the variables extracted from static posturography, which were all very close to normal values in the deep brain stimulation condition. These results suggest that non-dopaminergic pathways, such as those from the basal ganglia to brain stem centres, are probably involved in postural control. The effectiveness of deep brain stimulation, but not levodopa, in reducing postural sway is supported by studies showing that the former improved axial UPDRS measures and limb measures, ${ }^{3}$ but the latter did not improve axial measures. ${ }^{42}$ The action of deep brain stimulation may help integrate information from the proprioceptive system, improving kinaesthetic control of the centre of mass.

In the $d b s+d o p a$ condition, deep brain stimulation attenuated the negative effects on postural sway that levodopa introduced. ${ }^{3}$ In particular, mediolateral oscillation was reduced, suggesting more trunk stability. Nevertheless, the large sway area in the $d b s+d o p a$ condition showed that improved postural control caused by deep brain stimulation was not enough to compensate for the reduced stiffness of muscle and joints caused by levodopa. This linear summation of the effects from levodopa and deep brain stimulation has been observed previously in studies of changes in UPDRS measures, especially with globus pallidus stimulation. ${ }^{43}$

The asymmetrical nature of Parkinson's disease was reflected in postural sway variables extracted from each foot. For subjects with Parkinson's disease, results showed a large asymmetry for CoP mean velocity and $\mathrm{f}_{95 \%}$, but normal symmetry for rms. Thus the variables related more to neural control activity (mean velocity and $\mathrm{f}_{95 \%}$ ) present more differences between the feet in subjects with Parkinson's disease than the variable related to achievement of postural stability (rms), suggesting that symmetrical balance can be achieved through asymmetrical neural control activity. The improved symmetry of postural control between the legs in the $d b s$ condition also suggests that bilateral stimulation in the globus pallidus or subthalamic nucleus can normalise bilateral involvement of the legs for posture. In contrast, levodopa worsened asymmetry of postural control, perhaps because it decreased stiffness without improving postural control, thus revealing the underlying asymmetry.

The correlation analysis between each quantitative postural sway measure and the UPDRS motor examination showed that only $\mathrm{f}_{95 \%}$ of the CoP was significantly correlated with the UPDRS total score and the tremor and posture + gait subcomponents. The positive correlation between $\mathrm{f}_{95 \%}$ and clinical tremor measures showed that tremor influences postural stability and can be detected by static posturography. Even small amounts of tremor may be transmitted to forces at the ground and can therefore influence initiation and control of postural movements. The high correlation of $\mathrm{f}_{95 \%}$ with the posture+gait item primarily came from correlations with posture, gait, and bradykinesia (items 28,29, and 31). The significant correlations with gait and bradykinesia suggest that difficulties and slowness in motion can be paradoxically accompanied by high frequency components in body sway. In fact, the necessity for frequent neural control over the CoP displacement, rather than tremor per se, may be the cause of the difficulties and slowness in motion in subjects with Parkinson's disease. Poor correlations among the UPDRS scores, rms, and mean velocity suggest that UPDRS predicts sway area and velocity poorly.

\section{Conclusions}

Although correlations between UPDRS and sway variables may have been low in this study owing to the small number of subjects, clinicians might find it useful to measure the sway path, as rms has been related to the effectiveness of the postural control system, and mean velocity of sway has been related to the amount of postural regulatory activity. ${ }^{20}$ Results from this preliminary study suggest that quantitative evaluation of static posturography is sensitive to Parkinson's disease and to treatments with levodopa and deep brain stimulation, and such evaluation may become a useful adjunct to clinical measures of motor control in patients with Parkinson's disease.

\section{ACKNOWLEDGEMENTS}

This research was supported by NIH grants AG 40812 and AG 06457. We thank Dr K Burchiel and Dr V Anderson for patient referrals, Professor A Cappello for helpful reviewing, and A Gross for help with data collection.

\section{Authors' affiliations}

L Rocchi, L Chiari, Department of Electronics, Computer Science and Systems, University of Bologna, Bologna, Italy

F B Horak, Neurological Sciences Institute, Oregon Health \& Science University, Beaverton, Oregon, USA

Competing interests: none declared.

\section{REFERENCES}

1 Klawans HL. Individual manifestations of Parkinson's disease after ten or more years of levodopa. Mov Disord 1986;1:187-92.

2 Bonnet AM, Loria Y, Saint-Hilaire MH, et al. Does long-term aggravation of Parkinson's disease result from nondopaminergic lesions? Neurology 1987; 37: 1539-42.

3 Beijani BP, Gervais D, Arnulf I, et al. Axial Parkinsonian symptoms can be improved: the role of levodopa and bilateral subthalamic stimulation. J Neurol Neurosurg Psychiatry 2000;68:595-600.

4 Agid Y. Parkinson's disease: pathophysiology. Lancet 1991:337:1321-4.

5 Limousin $P$, Krack $P$, Pollak $P$, et al. Electrical stimulation of the STN in advanced Parkinson's disease. N Engl J Med 1998;339:1 105-1 1.

6 Prochazka A, Bennett DJ, Stephens M, et al. Measurement of rigidity in Parkinson's disease. Mov Disord 1997;12:4-32.

7 Schultz AB. Mobility impairment in the elderly: challenges for biomechanics research. J Biomech 1992;25:519-28.

8 Bloem BR, Beckley DJ, van Hilten BJ, et al. Clinimetrics of postural instability in Parkinson's disease. J Neurol 1998;245:669-73

9 Schieppati M, Nardone A. Free and supported stance in Parkinson's disease. The effect of posture and "postural set" on leg muscle responses to perturbation, and its relation to the severity of the disease. Brain $1991 ; 114: 1227-44$.

10 Horak FB, Nutt JG, Nashner LM. Postural inflexibility in Parkinsonian subjects. J Neurol Sci 1992;111:46-58.

11 Burleigh A, Horak FB, Nutt J, et al. Levodopa reduces muscle tone and lower extremity tremor in Parkinson's disease. Can J Neurol Sci 1995;22:280-5.

12 Mitchell SL, Collins JJ, De Luca CJ, et al. Open-loop and closed-loop postural control mechanisms in Parkinson's disease: increased mediolateral activity during quiet standing. Neurosci Lett 1995;197:133-6.

13 Maki BE, Mcllroy WE. Postural control in the older adult. Clin Geriatr Med 1996; 12:635-58.

14 Maki BE, Holliday PJ, Topper AK. A prospective study of postural balance and risk of falling in an ambulatory and independent elderly population. J Gerontol 1994;49:M72-84.

15 Frank J, Horak F, Nutt J. Centrally-initiated postural adjustments in Parkinsonian patients on and off levodopa. J Neurophysiol 2000;84:2440-48.

16 Horak F, Frank J, Nutt J. Effects of dopamine on postural control in Parkinsonian subjects: scaling, set, and tone. J Neurophysiol 1996;75:2380-96.

17 Robertson LT, Horak FB, Anderson VC, et al. Assessments of axial motor control during deep brain stimulation in Parkinsonian patients. Neurosurgery 2001;48:544-51.

18 Burchiel KJ, Anderson VC, Favre J, et al. Comparison of pallidal and subthalamic nucleus deep brain stimulation for advanced Parkinson's disease: results of a randomized, blinded pilot study. Neurosurgery 1999:45: 1375-82.

19 Winter DA. Human balance and posture control during standing and walking. Gait Posture 1995;3:193-214.

20 Prieto TE, Myklebust JB, Hoffmann RG, et al. Measures of postural steadiness: differences between healthy young and elderly adults. IEEE Trans Biomed Eng 1996;43:956-66.

21 Chiari L, Bertani A, Cappello A. Classification of visual strategies in human postural control by stochastic parameters. Hum Mov Sci 2000;19:817-42.

22 Prieto TE, Myklebust JB, Myklebust BM. Characterization and modeling of postural steadiness in the elderly: a review. IEEE Trans Rehab Eng $1993 ; 1: 26-34$

23 White R, Agouris I, Selbie RD, et al. The variability of force platform data in normal and cerebral palsy gait. Clin Biomech 1999;14:185-92. 24 Hintze JL. NCSS user's guide II. Kaysville, Utah: NCSS, 2000.

25 Yarrow K, Brown P, Gresty MA, et al. Force platform recordings in the diagnosis of primary orthostatic tremor. Gait Posture $2001 ; 13: 27-34$. 
26 Dietz V, Ziilstra W, Assaiante C et al. Balance control in Parkinson's disease. Gait Posture 1993;1:77-84.

27 Burleigh AL, Horak FB, Burchiel KJ, et al. Effects of thalamic stimulation on tremor, balance, and step-initiation: a single subject study. Mov Disord 1993:8:519-24.

28 Klockgether T, Borutta $M$, Rapp $H$, et al. A defect of kinesthesia in Parkinson's disease. Mov Disord 1995; 10:460-5.

29 Khudados E, Cody FW, O'Boyle DJ. Proprioceptive regulation of voluntary ankle movements, demonstrated using muscle vibration, is impaired by Parkinson's disease. J Neurol Neurosurg Psychiatry 1999:67:504-10.

30 Rickards C, Cody FW. Proprioceptive control of wrist movements in Parkinson's disease. Reduced muscle vibration-induced errors. Brain 1997; 120:977-90

31 Jobst EE, Melnick ME, Byl NN, et al. Sensory perception in Parkinson disease. Arch Neurol 1997;54:450-4.

32 Horak FB, Macpherson JM. Postural orientation and equilibrium. In: Shepard J, Rowell L, eds. Handbook of physiology: section 12. Exercise: regulation and integration of multiple systems. New York: Oxford University Press, 1996:255-92.

33 Dietz V, Gollhofer A, Kleiber M, et al. Regulation of bipedal stance: dependency on "load" receptors. Exp Brain Res 1992;89:229-31.

34 Maurer C, Mergner T, Bolha B, et al. Human balance control during cutaneous stimulation of the plantar soles. Neurosci Lett 2001;302:45-8.
35 Dickstein R, Shupert C, Horak F, et al. Fingertip touch improves postural stability in patients with peripheral neuropathy. Gait Posture $2001 ; 14: 238-47$

36 Horak F. Postural ataxia related to somatosensory loss. In: Rozicka E, Hallett M, Jankovix J, eds. Gait disorders. Advances in neurology, vol 87. Philadelphia: Lippincott Williams \& Wilkins, 2001:173-81.

37 Horak FB, Lamarre Y, Macpherson J, et al. Postural control in a patient with total body somatosensory loss [abstract] Soc Neurosci Abstr 1996;22:1632.

38 Roberts Warrior D, Overby A, Jankovic J, et al. Postural control in Parkinson's disease after unilateral posteroventral pallidotomy. Brain 2000;123:2141-9.

39 Marsden CD. Parkinson's disease. J Neurol Neurosurg Psychiatry 1994:57:672-81.

40 Jankovic J, McDermott M, Carter J, et al. Variable expression of Parkinson's disease: a base-line analysis of the DATATOP cohort. The Parkinson Study Group. Neurology 1990;40: 1529-34.

41 Mackinnon CD, Winter DA. Control of whole body balance in the frontal plane during human walking. J Biomech 1993;26:633-44.

42 Steiger MJ, Thompson PD, Marsden CD. Disordered axial movement in Parkinson's disease. J Neurol Neurosurg Psychiatry 1996;61:645-8.

43 Krack P, Pollak P, Limousin P, et al. Subthalamic nucleus or internal pallidal stimulation in young onset Parkinson's disease. Brain 1998:121:451-7.

\section{New JNNP online submission and review system}

The Editors of JNNP are pleased to inform authors and reviewers of its new online submission and review system. Bench $>$ Press is a fully integrated electronic system which uses the internet to allow rapid and efficient submission of manuscripts, plus the entire peer review process to be conducted online.

Authors can submit their manuscript in any standard word processing software. Graphic formats acceptable are: .jpg, .tiff, .gif, and eps. Text and graphic files are automatically converted to PDF for ease of distribution and reviewing purposes. Authors are asked to approve their submission before it formally enters the reviewing process.

To access the system click on "SUBMIT YOUR MANUSCRIPT HERE" on the JNNP homepage: http://www.jnnp.com/, or you can access Bench>Press directly at http://submit-innp.bmijournals.com/.

We are very excited with this new development and we would encourage authors and reviewers to use the on-line system where possible. It really is simple to use and should be a big improvement on the current peer review process. Full instructions can be found on Bench>Press http://submit-innp.bmijournals.com/, and JNNP online at http://www. jnnp.com/. Please contact Natalie Davies, Project Manager, ndavies@bmigroup.com for further information.

\section{PRE-REGISTER WITH THE SYSTEM}

We would be grateful if all JNNP authors and reviewers pre-registered with the system. This will give you the opportunity to update your contact and expertise data, allowing us to provide you with a more efficient service.

\section{Instructions for registering}

1. Enter http://submit-jnnp.bmijournals.com

2. Click on "Create a New Account" in the upper left hand side of the Bench>Press homepage

3. Enter your email address in the space provided.

4. Please be sure to enter the email address that you received this email message at.

You will be given the opportunity to change this once you have created an account.

5. Choose a password for yourself and enter it in the spaces provided.

6. Complete the question of your choice to be used in the event you cannot remember your password at a later time.

7. Click on the "Save" button at the bottom of the screen.

8. Check the e-mail account you registered under. An email will be sent to you with a verification number and URL.

9. Once you receive this verification number, click on the URL hyperlink and enter the verification number in the relevant field. This is for security reasons and to check that your account is not being used fraudulently.

10. Enter/amend your contact information, and update your expertise data. 